\title{
Protecting surface and buried structures from tunnelling using pile walls: a prediction model
}
A. Franza*
N. Losacco $^{\dagger}$
A. Ledesma $a^{\ddagger}$
G. M.B. Viggiani ${ }^{\S}$
R. Jimenez $\mathbb{I}$

\begin{abstract}
When tunnelling poses excessive risks for buildings and buried foundations, a pile row barrier may shield the existing structure from ground movements. This paper presents a three-dimensional linear elastic prediction method to evaluate the protective action of pile walls against surface and subsurface ground movements due to new tunnels, both directly behind the wall as well as within the entire ground. Analyses are carried out to evaluate the vertical and horizontal movements of the ground and the pile wall as the result of soil-pile row interaction. New factors that quantify the wall efficiency in reducing settlements and deflections behind the wall are proposed; the results indicate that the effectiveness of the pile wall at reducing horizontal displacements is limited. Subsequently, predictions are compared against field and numerical data to demonstrate that the elastic solution is applicable, particularly for small ground losses. Finally, the barrier efficiency in reducing settlements is discussed comparing pile walls and diaphragm walls.
\end{abstract}

Keywords: Tunnelling, settlement, pile, soil/structure interaction

\footnotetext{
*ETSI Caminos Canales y Puertos, Universidad Politécnica de Madrid, Madrid, Spain. Email: andreafranza@gmail.com.

${ }^{\dagger}$ Dept. of Civil, Environmental, Land, Building Engineering and Chemistry, Polytechnic University of Bari, Bari, Italy.

${ }^{\ddagger}$ Dept. of Civil and Environmental Engineering, Universitat Politècnica de Catalunya and International Centre for Numerical Methods in Engineering, Barcelona, Spain.

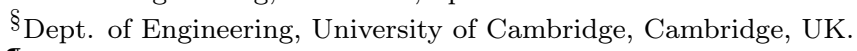

IETSI Caminos Canales y Puertos, Universidad Politécnica de Madrid, Madrid, Spain.
} 


\section{Introduction}

Engineers need to estimate the effects of tunnelling-induced ground movements on surface buildings, monuments, deep foundations and infrastructure as the result of tunnel-structure interaction (TSI). Figure 1 shows an example distribution of tunnelling-induced displacements in greenfield conditions (GF), when no structures are present. Typically, for preliminary assessments of the category of likely damage, a staged approach is used in which greenfield ground movements are estimated first and subsequently the TSI caused by the greenfield movements is addressed. Tensile strains in the structure under examination can be inferred directly from GF movements (Mair et al., 1996), using modification factors applied to the GF deformation parameters (Potts and Addenbrooke, 1997), or with two-stage interaction models using GF movements as input (Franza et al., 2020). Whatever the approach, an accurate prediction of greenfield ground movements is key for risk assessments.

When the risk predicted from the preliminary assessment is not deemed acceptable, mitigation actions must be implemented. Among others, a common protective measure against tunnelling-induced damage is the construction of embedded barriers (continuous diaphragm walls or rows of piles/micropiles) between the tunnel and the structure (Harris, 2001). The consequent tunnel-barrier interaction (TBI) results in mitigated ground movements for the TSI with respect to GF conditions. The efficacy of embedded barriers raising from the TBI problem has been addressed in numerical (Bilotta and Russo, 2011; Rampello et al., 2019), centrifuge (Song, 2019; Bilotta et al., 2006), and analytical studies (Ledesma and Alonso, 2017). However, previous empirical/analytical works (Ledesma and Alonso, 2017; Bilotta and Russo, 2011) focused on the effect of TBI on surface settlements. Thus, there is a lack of tools for a quick estimate of TBI subsurface vertical and horizontal ground movements, without the need for complex numerical models.

This paper aims to fill this gap by describing how an elastic 3D solution can be used 
to evaluate the effect of pile wall barriers on TBI movements while considering the barrier stiffness and geometry as well as the surface and subsurface GF ground input. The considered problem of piles/micropiles walls is described in Figure 2. In particular, a continuum-based two-stage model is used to solve the TBI and, then, to compute resulting displacements both at the wall location and within the ground. The wall mitigating effects are efficiently quantified by describing the problem geometry with respect to the tunnel depth and, thus, dimensionless results can be developed to be used in design. Finally, the proposed solution is compared with numerical and real data of pile and diaphragm walls, considering the predicted efficiency in reducing settlements as a function of the pile length-to-tunnel depth ratio.

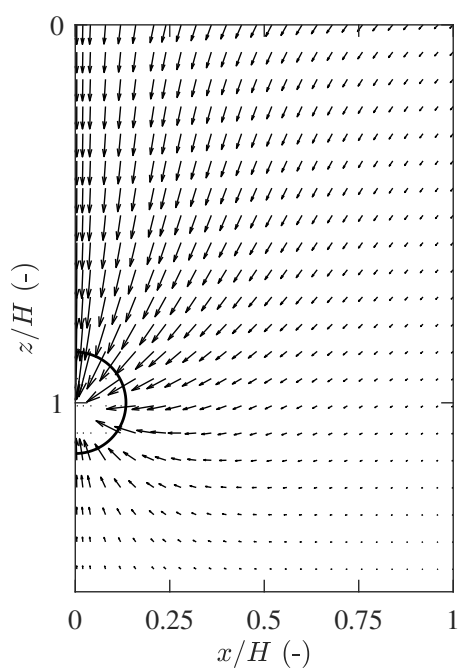

Figure 1. Tunnelling-induced displacements in the $\mathrm{x}-\mathrm{z}$ plane: greenfield (GF) semi-analytical distribution from Loganathan and Poulos (1998).

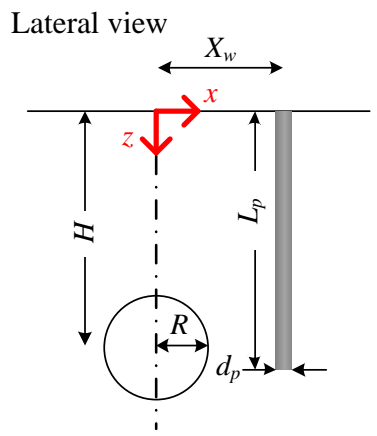

Top view

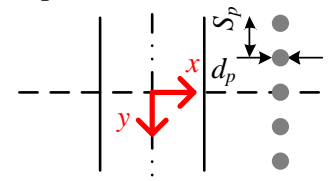

Figure 2. Considered problem of the tunnel-barrier interaction (TBI) and used notation.

\section{Greenfield displacement fields}

The interaction mechanisms with the wall depend on the greenfield displacement distribution and, if a linear elastic model with a perfect compatibility condition at the soil-wall interface 
is adopted, model outcomes are directly proportional to the tunnel ground loss. Therefore, it is important to analyse the shape associated with typical tunnelling-induced displacement fields. To this end, empirical and (semi-) analytical methods can be employed, as described next.

\subsection{Empirical methods}

In empirical approaches, greenfield tunnelling-induced settlements $u_{z}$ are usually described using a standard Gaussian distribution, while the horizontal movements $u_{x}$ are related to $u_{z}$ by assuming that the ground displacement vectors point towards a specific point, either coinciding with or below the tunnel axis (Mair and Taylor, 1997). The resulting equations, widely adopted in practice, are:

$$
\begin{array}{rlr}
u_{z}^{e m p}=u_{z, \text { max }} \exp \left[-\frac{x^{2}}{2 i^{2}}\right] & \text { in which } \frac{i}{H}=K_{s}+\frac{\partial i}{\partial z} \frac{z}{H} \\
u_{x}^{e m p}=\frac{x}{(H-z)} u_{z} & \text { vector focus at tunnel axis } \\
u_{x}^{e m p}=\frac{x}{(1+0.175 / 0.325) H} u_{z} & \text { vector focus deeper than tunnel axis }
\end{array}
$$

where $x$ and $z$ are the spatial coordinates (see Figure 2), $u_{z, \max }=\Delta V /(\sqrt{2 \pi} i)$ is the maximum settlement at the centreline (proportional to the ground loss $\Delta V$ ), and $i$ is the horizontal distance from the tunnel centreline to the inflection point of the curve. The value of this offset $i$ at depth, which controls the shape of the displacement field, can be computed using its surface value $\left(K_{s}\right)$ and its slope with depth $(\partial i / \partial z)$. For clays, Mair et al. (1993) suggested $K_{s}=0.5$ and $(\partial i / \partial z)=-0.325$; for sands and gravels, Mair and Taylor (1997) reported a significant data scatter for $K_{s}$, with values ranging from 0.25 to 0.45 . In practice, the ground loss is often normalised, resulting in the tunnel volume loss (in percentage) $V_{l, t}=\frac{\Delta V}{V_{0}} \times 100$, where $V_{0}$ is the theoretical tunnel volume. 
Normalising the spatial coordinates by the tunnel depth $H$, it is obtained that the shape of the displacement field is not affected by the tunnel diameter $D$; i.e., we have:

$$
\begin{aligned}
& u_{z}^{e m p}=u_{z, \max } \exp \left[-\frac{x^{2}}{2\left(K_{s}+\frac{\partial i}{\partial z} z^{\prime}\right)^{2}}\right] \\
& u_{x}^{e m p}=\frac{x^{\prime}}{\left(1-z^{\prime}\right)} u_{z} \\
& u_{x}^{e m p}=\frac{x^{\prime}}{(1+0.175 / 0.325)} u_{z}
\end{aligned}
$$

vector towards point deeper than tunnel axis

vector towards tunnel axis

where the prime $\left(^{\prime}\right)$ denotes normalisation by $H\left(\right.$ e.g. $\left.x^{\prime}=x / H\right)$.

\subsection{Analytical solutions}

Analytical solutions relate tunnelling displacements to a displacement field at the tunnel periphery. Assuming an infinite space (ideal for deep tunnels), Pender (1980) provided the displacements induced by a tunnel in an elastic medium with anisotropic initial stresses: volumetric stress relief produces a uniform convergence of the tunnel periphery, while deviatoric stress changes induce an ovalization. These uniform convergence and ovalization displacements may be normalised by the tunnel radius, $R$, to define the tunnel deformation components $\varepsilon$ and $\delta$, respectively. As an alternative, the relative distortion parameter $\rho=\delta / \varepsilon$ can be used.

The superposition of singularities method may also be used to estimate tunnellinginduced ground displacements within a linear elastic half-space. Sagaseta (1987) evaluated movements assuming that a void (at depth $H$ and of volume equal to the tunnel ground loss, $\Delta V$ ) is filled by the surrounding incompressible, linear elastic, and isotropic soil. Then, Verruijt and Booker (1996) used Pender's equations with the superposition of singularities 
method to account for convergence and ovalization, quantified by $\varepsilon$ and $\delta$, which are regarded as input parameters. For a small tunnel convergence, $\frac{\Delta V}{V_{0}} \approx 2 \varepsilon$ and, thus, the tunnel volume $\operatorname{loss} V_{l, t} \approx 200 \varepsilon$

By rearranging Verruijt and Booker (1996) and González and Sagaseta (2001) solutions, horizontal $\left(u_{x}\right)$ and vertical $\left(u_{z}\right)$ displacements for an incompressible elastic medium (Poisson's ratio $\left.\nu_{s}=0.5\right)$ can be obtained in a normalised form. They are:

$$
\begin{aligned}
& \frac{u_{x}^{e l}}{u_{\text {norm }}}=-\frac{x^{\prime}}{2{r^{\prime}}_{1}^{2}}\left(1-\rho \frac{x^{\prime 2}-z_{1}^{\prime 2}}{r_{1}^{\prime 2}}\right)-\frac{x^{\prime}}{2{r_{2}^{\prime}}_{2}^{2}}\left(1-\rho \frac{x^{\prime 2}-z_{2}^{\prime 2}}{r_{2}^{\prime 2}}\right)+\frac{4 x^{\prime} z^{\prime}}{2{r_{2}^{\prime}}_{2}^{2}}\left(\frac{z_{2}^{\prime}}{r_{2}^{\prime 2}}-\rho \frac{x^{\prime 2}-3{z^{\prime}}_{2}^{2}}{r_{2}^{\prime 4}}\right) \\
& \frac{u_{z}^{e l}}{u_{\text {norm }}}=-\frac{z_{1}^{\prime}}{2{r^{\prime}}_{1}^{2}}\left(1-\rho \frac{x^{\prime 2}-z_{1}^{\prime 2}}{{r^{\prime}}_{1}^{2}}\right)+\frac{z_{2}^{\prime}}{2{r^{\prime}}_{2}^{2}}\left(1+\rho \frac{x^{\prime 2}-{z^{\prime}}_{2}^{2}}{r_{2}^{\prime 2}}\right) \\
& -\frac{1}{2{r_{2}^{\prime}}_{2}^{2}}\left(2\left(z^{\prime}+\rho\right) \frac{x^{\prime 2}-z_{2}^{\prime 2}}{r_{2}^{\prime 2}}+4 \rho z^{\prime} z_{2}^{\prime} \frac{3{x^{\prime}}^{2}-z_{2}^{\prime 2}}{r_{2}^{\prime 4}}\right)
\end{aligned}
$$

where $u_{\text {norm }}$ is a scalar proportional to the ground loss $\Delta V$ as shown Equation (4)

$$
u_{\text {norm }}=2 \varepsilon R\left(\frac{R}{H}\right)=2 u_{\varepsilon}\left(\frac{R}{H}\right)=\frac{\Delta V}{\pi H}
$$

and $z_{1}=z-H, z_{2}=z+H$, and $r_{1}=\sqrt{x^{2}+(z-H)^{2}}$. Note, therefore, that the right terms of Equation (3) describe the shape of the displacement field, while the left terms are the normalised displacements. As in Equation (2), the shape of the elastic displacement field in Equation (3) is not affected by the tunnel size, while its amplitude is directly proportional to the tunnel ground loss.

The robustness of analytical solutions was illustrated by Pinto et al. (2014) through comparison with case studies, while Pinto and Whittle (2014) developed closed formulas to consider the heading advancement by integrating volume losses, and the resulting threedimensional displacement field. 


\subsection{Semi-analytical formulas}

Semi-analytical solutions, $u^{s a}$, have been developed by applying an empirical corrective term, $\xi$, to elastic displacement patterns, $u^{e l}$. Loganathan and Poulos (1998) proposed evaluating vertical and horizontal undrained movements in clays correcting the elastic solution of Sagaseta (1987) (i.e. Equation (3) for $\delta=0$ ) with the term in Equation (5), chosen to account for field observations and centrifuge model test outcomes (Pinto and Whittle, 2006).

$$
u_{z}^{s a}=\xi u_{z}^{e l} ; \quad u_{x}^{s a}=\xi u_{x}^{e l} ; \quad \xi=2 \exp \left[-\left(\frac{1.38 x^{\prime 2}}{(1+R / H)^{2}}+0.69 z^{\prime 2}\right)\right]
$$

Differently than before, this $\xi$ correction makes the displacement field shape to depend on tunnel size, through its radius, $R$. However, Loganathan and Poulos (1998) reported that the inflection point offset for the semi-analytical field is $i=0.62 H^{0.9} R^{0.1}$ so that, for a tunnel radius between 2 and $8 \mathrm{~m}, R^{0.1}=1.07-1.23$. This relatively small range suggests that the semi-analytical displacement field is not highly dependent on $R$ (this point is further discussed in the paper). Assuming a representative value of $R^{0.1} \approx 1.16$, it follows that $i \approx 0.72 H^{0.9}$. Recently, Zhang et al. (2020) generalised the expression for the corrective factor in sandy soils to account for the head advancement.

\section{Tunnel-barrier interaction problem parameters}

To generalise the outcomes of the elastic solution, the TBI problem can be described in dimensionless form, hence reducing the number of variables involved. The output variables $\left(u_{z}, u_{x}\right)$ depend on the input non dimensional groups and on the tunnel volume loss, $V_{l, t}$. Two sets of dimensionless groups are analysed in this paper.

Group set $\Pi$ are consistent with Ledesma and Alonso (2017), and are also used to compare 
111

112

with their plane-strain results:

$$
\Pi_{1}=\frac{H}{R} \quad \Pi_{2}=\frac{X_{w}}{R} \quad \Pi_{3}=\frac{L_{p}}{R} \quad \Pi_{4}=\frac{E_{s} R S_{p}}{E_{p} A_{p}} \quad \Pi_{5}=\frac{E_{s} R^{3} S_{p}}{E_{p} I_{p}} \quad \Pi_{6}=\frac{S_{p}}{d_{p}}
$$

Group set $\Psi$ are suggested (for the first time in this paper) as an improved alternative, considering with respect to $H$ the marginal effect of $R$ on the shape of the displacement field affecting the soil-wall interaction:

$$
\Psi_{1}=\frac{H}{R} \quad \Psi_{2}=\frac{X_{w}}{H} \quad \Psi_{3}=\frac{L_{p}}{H} \quad \Psi_{4}=\frac{E_{s} H S_{p}}{E_{p} A_{p}} \quad \Psi_{5}=\frac{E_{s} H^{3} S_{p}}{E_{p} I_{p}} \quad \Psi_{6}=\frac{S_{p}}{d_{p}}
$$

In Equations (6) and (7), $X_{w}$ is the pile wall offset from the tunnel centreline; $L_{p}, S_{p}, d_{p}$ $A_{p}$ and $I_{p}$ are the length of embedment, the longitudinal spacing, the diameter, the crosssectional area and the second moment of area of the piles; and $E_{p}$ and $E_{s}$ are the Young's modulus of the pile and of the soil, respectively. Equation (8) specialises dimensionless groups $\Psi$ for piles with a circular solid cross-section, a frequent construction case:

$$
\Psi_{1}=\frac{H}{R} \quad \Psi_{2}=\frac{X_{w}}{H} \quad \Psi_{3}=\frac{L_{p}}{H} \quad \Psi_{4}=1.3 \Psi_{6} \frac{E_{s}}{E_{p}} \frac{H}{d_{p}} \quad \Psi_{5}=20.4 \Psi_{6} \frac{E_{s}}{E_{p}}\left(\frac{H}{d_{p}}\right)^{3} \quad \Psi_{6}=\frac{S_{p}}{d_{p}}
$$

The shape of the greenfield GF input is another important aspect that impacts the TBI interaction results. For brevity, all analyses in this paper were conducted using the greenfield displacement field from Loganathan and Poulos (1998), which is considered an effective approximation of a tunnelling-induced ground deformation mechanism. However, any greenfield input may be selected in the considered model.

The impact of the wall on the displacements is described using two dimensionless terms: the wall efficiency, $\eta_{w}$, that considers settlements at the wall location; and the local efficiency, $\eta_{l}$, that considers vertical and horizontal displacements over the entire ground 
(i.e. both surface and subsurface locations). They are defined as:

$$
\eta_{w}=1-\frac{u_{z, w}}{u_{z, w}^{G F}} \quad \eta_{l, z}=1-\left|\frac{u_{z, s}}{u_{z, s}^{G F}}\right| \quad \eta_{l, x}=1-\left|\frac{u_{x, s}}{u_{x, s}^{G F}}\right|
$$

where the superscript GF stands for greenfield, while the subscripts $w$ and $s$ stand for wall and soil, respectively. Note that efficiencies are defined using the absolute value of the ratio between displacements; this is to make them describe the normalised magnitude of the interaction displacement (rather than being affected by a change in movement direction between greenfield and interaction analyses). A positive efficiency $\left(\eta_{l}>0\right)$ indicates a decrease in the pile or local soil displacement with respect to the greenfield condition, whereas a negative efficiency $\left(\eta_{l}<0\right)$ occurs when the magnitude of movements increases due to the wall action.

Finally, the efficiency to reduce the wall curvature with respect to the greenfield horizontal displacement profile is defined as:

$$
\omega_{w}=1-\frac{\chi_{w}}{\chi_{w}^{G F}}
$$

where $\chi$ is the curvature (i.e., second derivative of the horizontal displacement profiles). This factor $\omega_{w}$ depends on the relative soil-pile bending stiffness, and quantifies the barrier action to prevent flexural distortions of structures buried close to the barrier.

\section{Model}

In this paper, a linear elastic continuum-based two-stage analysis model is adopted (Franza et al., 2019b,a) to analyse the three-dimensional response of a pile row with free heads and aligned with the tunnel longitudinal direction (as sketched in Figure 2). Each pile is modelled as a vertical beam embedded into the elastic half-space subjected to steady-state 
147

tunnelling-induced ground movements; thus, the soil response in the three directions $(x$, $y, z)$ is fully coupled while for the pile axes vertical, horizontal, and rotational degrees of freedom are considered. This approach is consistent with previous works considering tunnelpile group interaction analyses (Basile, 2014; Chen et al., 1999; Loganathan et al., 2001). The excavation is considered using its induced greenfield ground movements and an equivalent set of forces capable to induce these greenfield movements in the absence of piles, while the soil response to loading is not affected by the presence of the excavation. The soil is modelled as a homogeneous and isotropic half-space (referred to as a continuum) with a perfect compatibility condition at the soil-pile interface. The soil flexibility along the pile is obtained by integrating Mindlin's solutions, while Mindlin's formulas directly provide the displacement field of the remaining ground.

The three-dimensional elastic solution (EL 3D) was obtained using the Finite Element method solving the equilibrium Equation (11). This equation provides the pile wall displacements $\mathbf{u}_{w}$ for the barrier-soil system subjected to the tunnelling-induced equivalent forces, which induce the tunnelling-induced displacements at the wall locations for no barrier. Then, the vector of the forces applied by the pile group to the soil continuum in the tunnellinginduced equilibrium condition are inferred from Equation (12), which was obtained from the principle of action and reaction (forces applied by the piles to the soil opposite to the forces applied by the soil to the piles). Alternatively, this force vector may be also expressed as the forces needed to displace the continuum of the difference between the soil displacements in presence of the barrier and the greenfield soil displacements. Once the interaction forces applied along the pile axes are known, Equation (13) gives the post-tunnelling displacement field within the entire half-space $\mathbf{U}_{s}$, summing the ground interaction displacement (propagated from the pile to the entire ground) and the greenfield displacement field. That 
172

$$
\left(\mathbf{k}_{w}+\mathbf{k}_{\mathbf{s}}\right) \mathbf{u}_{w}=\mathbf{k}_{\mathbf{s}} \mathbf{u}_{w}^{g f}
$$

$$
\mathbf{f}_{w}=-\mathbf{k}_{w} \mathbf{u}_{w}=\mathbf{k}_{\mathbf{s}}\left(\mathbf{u}_{w}-\mathbf{u}_{w}^{g f}\right)
$$

173

$$
\mathbf{U}_{s}=\mathbf{U}_{\mathbf{s}}{ }^{i n t}+\mathbf{U}_{\mathbf{s}}{ }^{g f} ; \quad \mathbf{U}_{\mathbf{s}}{ }^{i n t}=\mathbf{L}_{\mathbf{s}} \mathbf{F}_{w}
$$

174

where $\mathbf{u}_{w}$ is the displacement vector of the pile group; $\mathbf{u}_{w}^{g f}$ is the greenfield displacement vector along the piles; $\mathbf{k}_{w}$ and $\mathbf{k}_{s}$ are, respectively, the stiffness matrix of the pile group and the ground (at the pile axis nodes); $\mathbf{f}_{\mathrm{w}}$ is the vector of interaction forces applied by the pile nodes to the soil; $\mathbf{U}_{s}$ and $\mathbf{U}_{s}^{g f}$ are the final and greenfield displacements of the ground; $\mathbf{F}_{w}=\left[\mathbf{f}_{w}, 0,0 \ldots 0\right]^{T}$ is the vector of interaction forces applied to the ground; $\mathbf{L}_{s}$ is the soil flexibility matrix (giving the relationship between applied forces and displacements).

In other words, the model propagates within the half-space the displacement difference, at the pile location, between the tunnelling-induced displacements and the greenfield profiles. The final interaction displacement field is given by the superposition of the propagated difference in displacements at the pile location and the greenfield movements. In this paper, ground movements $\mathbf{U}_{s}$ within the transverse plane passing through the barrier centre are reported ( $y=0$ in Figure 2). 
186

187

\section{Parametric study}

\subsection{Considered scenarios}

Two tunnelling scenarios having a tunnel axis depth of $H=15 \mathrm{~m}$ are considered: 'Case A' with tunnel radius $R=5 \mathrm{~m}$ and a cover to diameter ratio $C / D=1$; 'Case $\mathbf{B}$ ' with $R=2.5 \mathrm{~m}$ and $C / D=2.5$. Both tunnels are constructed in a homogeneous soil with a Young's modulus $E_{s}=7.5 \mathrm{MPa}$ and a Poisson's ratio $\nu_{s}=0.5$. A protective wall is considered consisting of 11 bored concrete piles of varying length $L_{p}=10,20,30,40 \mathrm{~m}$ with a longitudinal spacing $S_{p}=2 \mathrm{~m}$, diameter $d_{p}=1.12 \mathrm{~m}$, and Young's modulus $E_{p}=30 \mathrm{GPa}$. Pile walls at two different transverse offsets from the tunnel centreline of $X_{w}=5,10 \mathrm{~m}$ are studied, so that the first is relatively close to the tunnel $\left(X_{w} / H=0.33 \approx 0.3\right)$ and the second is relatively far from it $\left(X_{w} / H=0.67 \approx 0.7\right)$. These offsets are referred to as 'close location' and 'far location', respectively. The normalised pile spacing for both Cases A and $\mathrm{B}$ is $S_{p} / d_{p}=1.79$, since that is close to frequent construction scenarios. This value also produces a nearly uniform displacement field within the vertical plane containing the piles, i.e. there is only a minor variation of displacements along the longitudinal direction $y$ (Bilotta and Russo, 2011).

Table 1 reports $\Pi$ and $\Psi$ values associated with all analysed scenarios. Selected pile lengths cover the scenario of wall tip above $\left(L_{p} / H \approx 0.7\right)$, adjacent $\left(L_{p} / H \approx 1.3\right)$, and below $\left(L_{p} / H \approx 2.7\right)$ the tunnel axis. More importantly, this choice of dimensionless groups and the adopted greenfield allows to: [i] compare results directly with the elastic settlement prediction of Ledesma and Alonso (2017) who considered a 2D scenario equivalent to Case A; [ii] evaluate the efficiency of dimensionless inputs $\Psi$ against $\Pi$, considering that Cases A and B have equal $\Psi$ groups (except for $\Psi_{1}$ whose impact is limited within the considered range).

Additionally to the simulations in Table 1, and to investigate the influence of the relative 
211

soil-to-pile stiffness, simulations were repeated, for the pile wall with $X_{w} / H \approx 0.7$, for several $E_{s} / E_{p}$ ratios and, thus, for an extended range of $\Psi_{4}$ and $\Psi_{5}$ values.

Table 1. Considered scenarios for both Case A $(C / D=1)$ and Case B $(C / D=2.5)$ : dimensionless groups.

\begin{tabular}{lllllll}
\hline & $\Pi_{1}$ & $\Pi_{2}$ & $\Pi_{3}$ & $\Pi_{4}$ & $\Pi_{5}$ & $\Pi_{6}$ \\
& $H / R$ & $X_{w} / R$ & $L_{p} / R$ & Axial & Bending & $S_{p} / d_{p}$ \\
Case A & 3 & 1,2 & $1,2,4,8$ & 0.0025 & 0.81 & 1.79 \\
Case B & 6 & 2,4 & $2,4,8,16$ & 0.0013 & 0.10 & 1.79 \\
\hline & $\Psi_{1}$ & $\Psi_{2}$ & $\Psi_{3}$ & $\Psi_{4}$ & $\Psi_{5}$ & $\Psi_{6}$ \\
& $H / R$ & $X_{w} / H$ & $L_{p} / H$ & Axial & Bending & $S_{p} / d_{p}$ \\
Case A & 3 & $0.3,0.7$ & $0.7,1.3,2,2.7$ & 0.0076 & 21.85 & 1.79 \\
Case B & 6 & $0.3,0.7$ & $0.7,1.3,2,2.7$ & 0.0076 & 21.85 & 1.79 \\
\hline
\end{tabular}

\subsection{Comparison with $2 \mathrm{D}$ elastic solutions}

For selected cases with a pile offset $X_{w} / H \approx 0.7$, Figure 3 shows surface settlements (Figure 3a) and the wall efficiency $\eta_{w}$ (Figure 3b) predicted from the proposed model (EL 3D), along with 2D elastic (EL 2D) and numerical (NUM 2D) results from Ledesma and Alonso (2017).

Figure 3a displays a good qualitative agreement between the surface settlement profiles predicted by 3D EL and 2D EL models, for all relative pile-to-tunnel depth $L_{p} / H$. Also, $3 \mathrm{D}$ results confirmed previous works indicating that the barrier effect is negligible for the wall tip located above the tunnel $\left(L_{p} / H \approx 0.7\right)$, while it is significant for piles embedded below the tunnel level $\left(L_{p} / H>1\right)$. Importantly, the wall efficiency associated with the 3D model is lower than for 2D results, as quantified by Figure 3b. This is due to the proposed 3D EL model taking into account the pile spacing and the finite number of piles within the barrier. Therefore, for a conservative estimate of pile wall settlement reduction, the 3D solution should be used instead of the 2D model.

Next, the effects of the tunnel radius on the wall efficiency $\eta_{w}$ are discussed. Despite the use of the Loganathan and Poulos (1998) semi-analytical formula for the greenfield settle- 

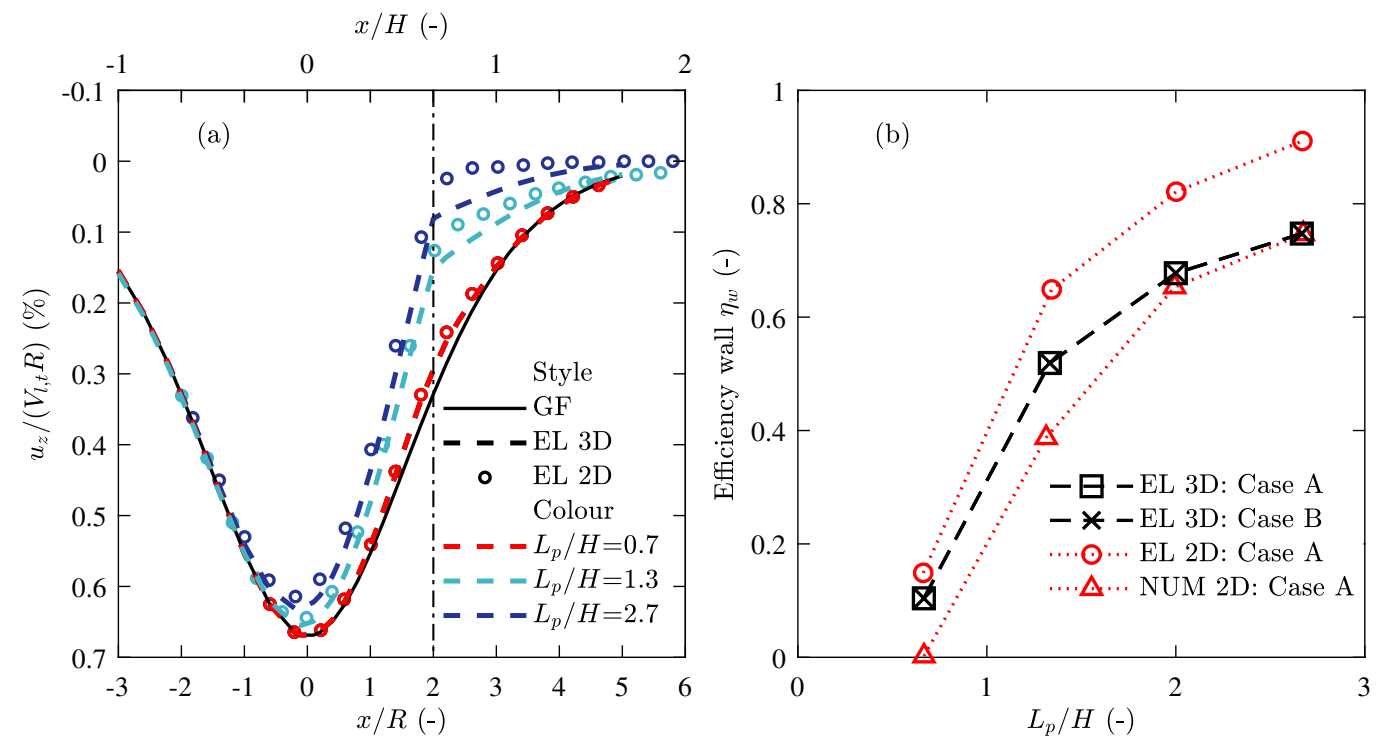

Figure 3. Comparison between 3D proposed model and 2D results of Ledesma and Alonso (2017) for varying wall length $L_{p} / H$ and pile offset $X_{w} / H=0.7$. (a) Surface settlements for Case A; (b) wall efficiency for Cases A and B.

ments (with a slight displacement shape dependence on $R / H$ ), the wall efficiency calculated by the EL 3D model are nearly identical in Figure 3b for Cases A and B, having different tunnel radius $R$ and, thus, $R / H$. Consequently, Figure $3 \mathrm{~b}$ demonstrates that the radius $R$ has a negligible role on the wall efficiency and, thus, the tunnel-wall interaction mechanism. Also, it supports the argument that the dimensionless groups $\Psi$ (defined with respect to the tunnel depth $H$ ) are more adequate than groups $\Pi$ (using the tunnel radius $R$ ). To support both statements, analyses for the pile walls with $X_{w} / H \approx 0.7$ and $L_{p} / H \approx 1.3$ in Cases $\mathrm{A}$ and B gave nearly identical surface and subsurface ground movements (see supplemental data in Figure S1).

\subsection{Influence of wall on surface and subsurface ground deformations}

In this section, the capabilities of the model to predict vertical and horizontal movements are studied, and the local efficiencies for the eight pile walls considered in the parametric 
study (close barrier $X_{w} / H \approx 0.3$, far barrier $X_{w} / H \approx 0.7$, and $L_{p} / H=0.7,1.3,2.0,2.7$ ). Although reported, ground settlements obtained from the relatively short walls with $L_{p} / H \approx$ 0.7 are close to greenfield conditions and, thus, they are not discussed.

Firstly, vertical and horizontal ground movements normalised by $u_{\text {norm }}$ at the surface $(z / H=0)$ and mid-depth $(z / H=0.5)$ are plotted in Figures $4 \mathrm{a}$ and $\mathrm{b}$, respectively. Interestingly, similar settlements are obtained at $x / H>0.7$, beyond the far wall location, for both pile wall offsets $\left(X_{w} / H \approx 0.3\right.$ and 0.7$)$. However, the close barrier $\left(X_{w} / H=0.3\right)$ also decreases the settlements directly above the tunnel, particularly near the surface, while this latter mechanism is negligible for the far barrier $\left(X_{w} / H=0.7\right)$.

Then, Figures 4c and e show, for both pile locations, the ground settlements along the control vertical sections located at the pile locations $\left(X_{w} / H=0.3\right.$ and 0.7$)$. Horizontal ground movements are smoothed by the pile wall bending stiffness at the wall location (when $x=X_{w}$ ), with the wall deflection shape depending on the greenfield ground distribution, which differs between $X_{w} / H=0.3$ and 0.7 . Interestingly, Figure 4e displays a dragging effect that the pile wall close to the tunnel applies on the ground at the far location (when $x \neq X_{w}$ ); this is due to the wall bending stiffness resulting in a smooth horizontal displacement profile at the pile location $\left(\mathbf{U}_{\mathbf{s}}{ }^{i n t}\right)$ that propagates from the piles (a source) within the half-space.

Similarly, ground settlements in Figures $4 \mathrm{~d}$ and $\mathrm{f}$ also indicate the high axial stiffening action of the barrier at the wall location for the pile tip below the tunnel (nearly preventing differential settlements at $x=X_{w}$ along the pile depth). While the difference between interaction and final ground settlements (propagated to the entire ground, see Equation (12)) contributed to the damping of the settlements at the far location of $x / H=0.7$ when the pile is at $X_{w} / H=0.3$, nearly negligible effects are induced by the far wall with $X_{w} / H=0.7$ on the control section at $x / H=0.3$. This is due to smaller greenfield settlements at $X_{w} / H=0.7$ and, as a consequence, a smaller difference in displacements between the pile settlements and the greenfield. 
It is also of interest to consider the vertical wall efficiency $\eta_{w}$ associated with the six pile configurations. When the pile tip is above the tunnel axis depth $\left(L_{p} / H=0.7\right)$ negative efficiency is obtained for the close pile location $X_{w} / H=0.3$ because the pile is within the tunnel influence, subjected to settlements increasing in magnitude with depth, and thus it settles slightly more than the greenfield trough at the surface (Franza et al., 2019b). On the other hand, although the ground movements from the pile wall (PW) analyses indicate that the wall offset $X_{w} / H$ has an impact on subsurface ground movements (while its effect on surface displacement beyond the wall is small), wall efficiency factors in Figure 5 are similar for the two wall offsets $\left(X_{w} / H=0.3\right.$ and 0.7$)$ when the wall tip is below the tunnel $\left(L_{p} / H>1\right)$, as obtained by Ledesma and Alonso (2017). Furthermore, the wall efficiency does not consider the change in the shape of the interaction displacements with respect to the greenfield. Thus, engineering judgement is needed during the design of mitigation barriers when the wall efficiency is used.

As an alternative to wall efficiency $\eta_{w}$ defined at the pile location, the local efficiency $\eta_{l}$ is a more appropriate way to evaluate the effectiveness of the barrier in decreasing the ground movements at a specific location, corresponding to critical surface or subsurface structures/foundations. For three selected pile walls scenarios $\left(X_{w} / H=0.3\right.$ and $L_{p} / H=1.3$, $X_{w} / H=0.7$ and $L_{p} / H=1.3, X_{w} / H=0.7$ and $\left.L_{p} / H=2.7\right)$, Figure 6 displays contours for post-tunnelling normalised movements $\left(u / u_{\text {norm }}\right)$ shielded by the pile walls. Settlements are on the left and horizontal movements are on the right. For the horizontal movements the absolute value is plotted, with movements being directed towards the tunnel, while upwards settlements are set equal to zero. Using a similar layout, Figure 7 reports contours of vertical and horizontal local efficiency $\left(\eta_{l}\right)$ giving the relative reduction in movement magnitude.

The effect of the pile normalised offset is described by the comparison of the sub-plots $\mathrm{a}$ and $\mathrm{b}$ of Figures 6 and 7 . These plots confirm that a similar vertical efficiency $\eta_{l, z}$ is obtained along the entire pile length. However, while the mitigating effect of the closer wall 

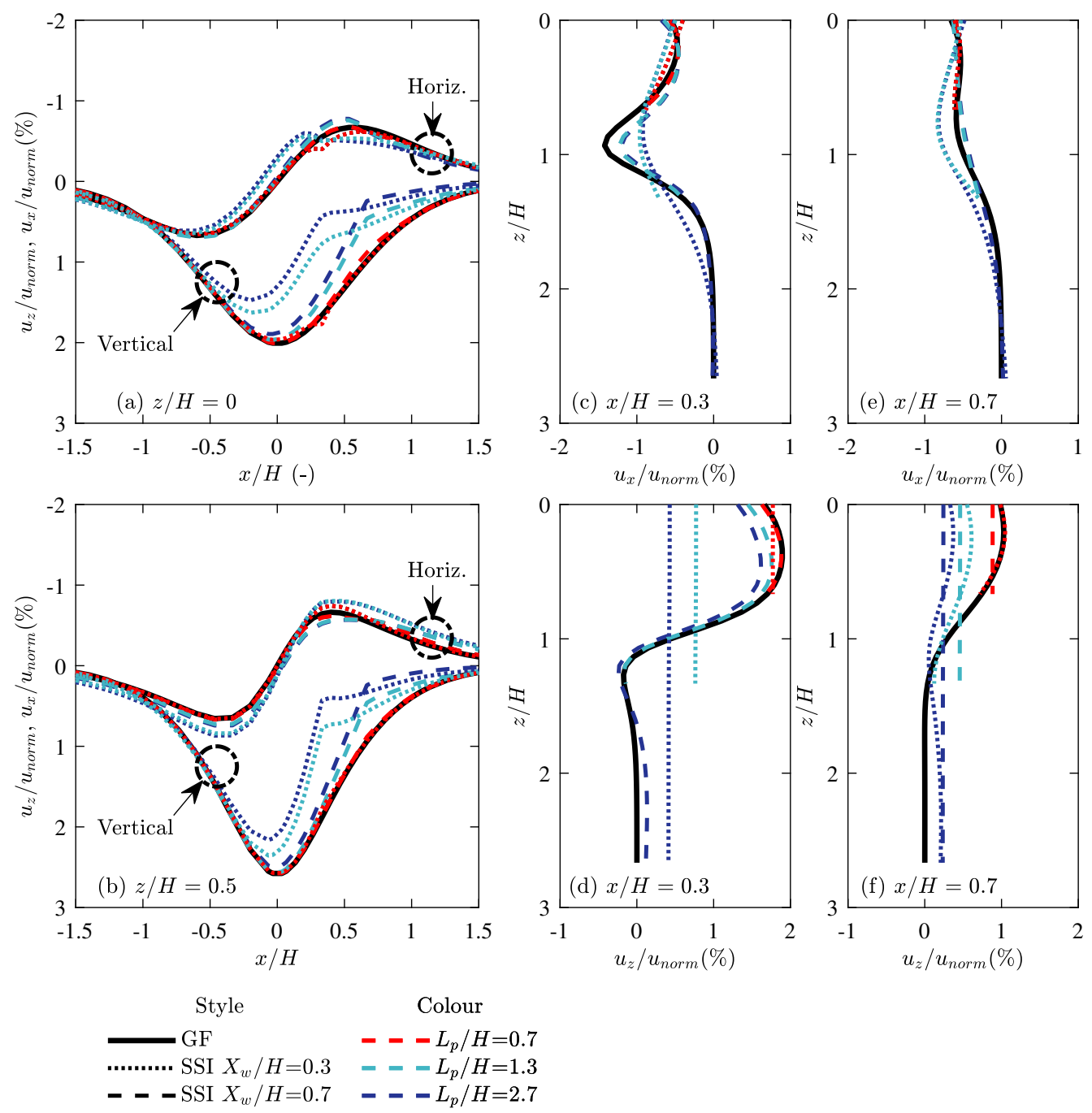

Figure 4. For different pile wall geometries $\left(X_{w} / H=0.3 ; 0.7\right.$ and $\left.L_{p} / H=0.7 ; 1.3 ; 2.7\right)$, vertical and horizontal ground movements along: (a) surface level; (b) subsurface level of $z / H=$ 0.5 ; (c-d) close vertical control section at $x / H=0.3$; (e-f) far vertical control section at $x / H=$ 0.7 . 


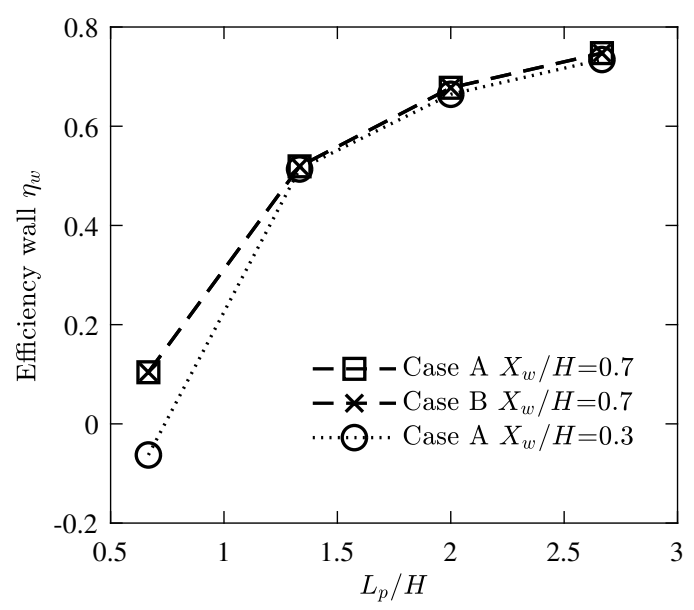

Figure 5. Wall efficiency for varying normalised pile length $L_{p} / H$.

$\left(X_{w} / H=0.3\right)$ extends also on the tunnel side, decreasing surface settlements above the centreline and subsurface settlements between $x / H=0.3-0.7$, the far pile $\left(X_{w} / H=0.7\right)$ only decreases settlements for $x / H=0.3-1.0$. In other words, for both offsets the wall decreases the settlements within an influence zone, extending at the surface between the relative distance of $\pm 0.5\left(x-X_{w}\right) / H$ from the wall. On the other hand, horizontally the pile wall leads to minor variations of $u_{x}$. The local efficiency $\eta_{l, x}$ has small positive values at the surface beyond the wall $\left(x>X_{w}\right)$ as well as at the subsurface between the tunnel centreline and the wall $\left(x=0\right.$ to $\left.X_{w}\right)$; however, negative values of $\eta_{l, x}$, associated with greater horizontal movements, are induced underneath the surface beyond the wall $\left(x>X_{w}\right)$.

Next, sub-plots $\mathrm{b}$ and $\mathrm{c}$ of Figures 6 and 7 show the minor influence of the pile embedment length $L_{p} / H$ on the ground horizontal movements $u_{x}$ and efficiency $\eta_{l, x}$, provided that the pile tip is below the tunnel depth. However, pile length does affect settlements, and there is a significant settlement reduction $u_{z}$ and increased local efficiency $\eta_{l, z}$ beyond the wall (i.e., for $x / H$ greater than $X_{w} / H$, that is 0.7 in these figures). As previously discussed, this is because of a greater portion of the pile being embedded in ground that is not affected by tunnelling. 


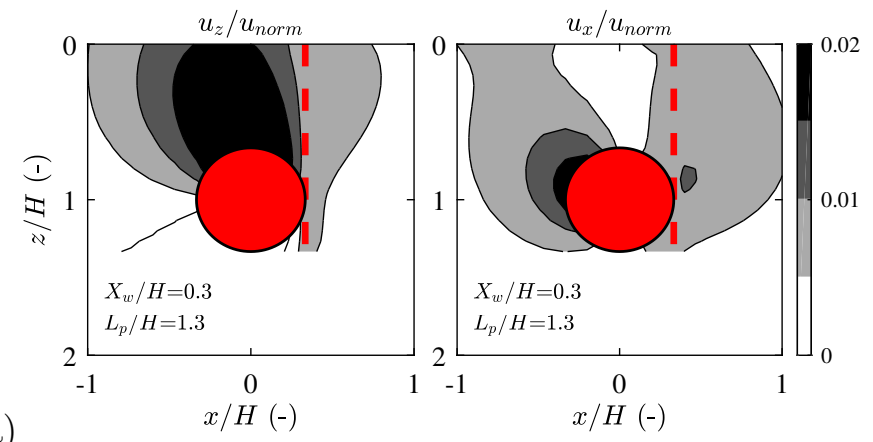

(a)
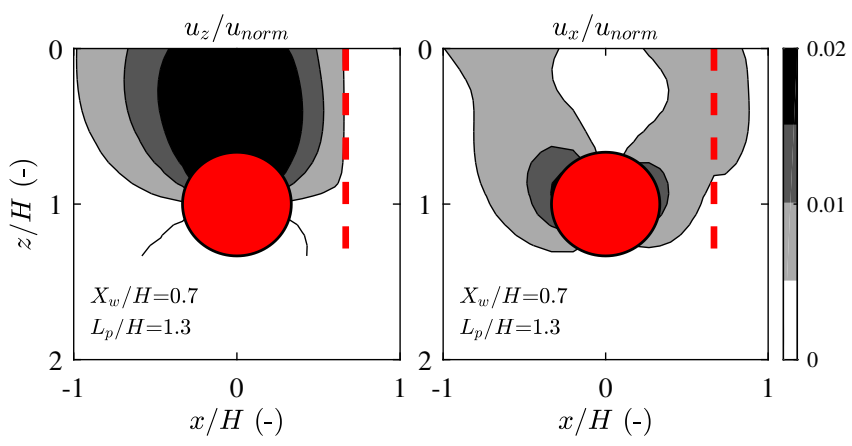

(b)
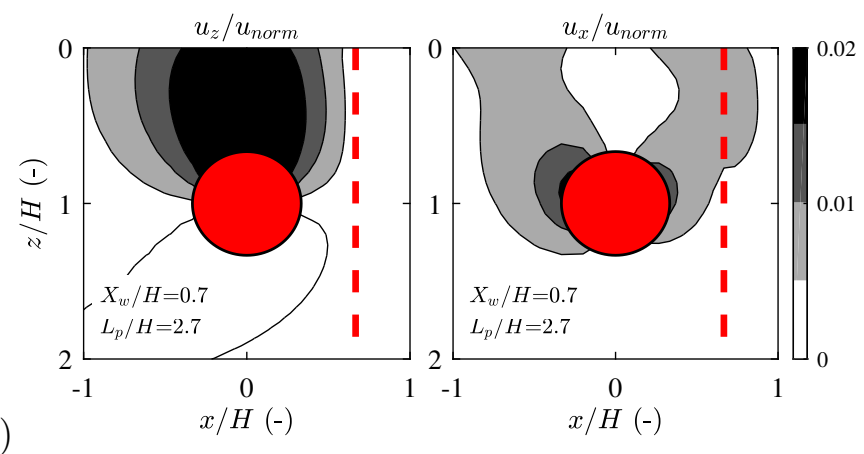

Figure 6. Vertical (left) and horizontal (right) normalised movements $u / u_{\text {norm }}$ : varying pile location (a vs b) and length (b vs c). 

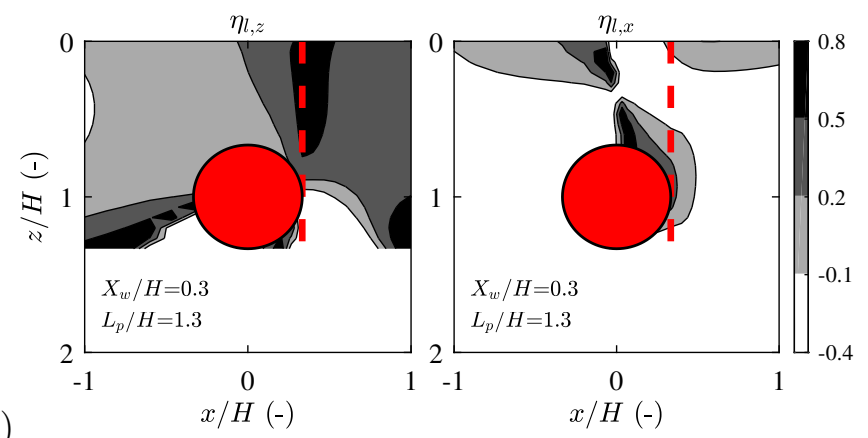

(a)
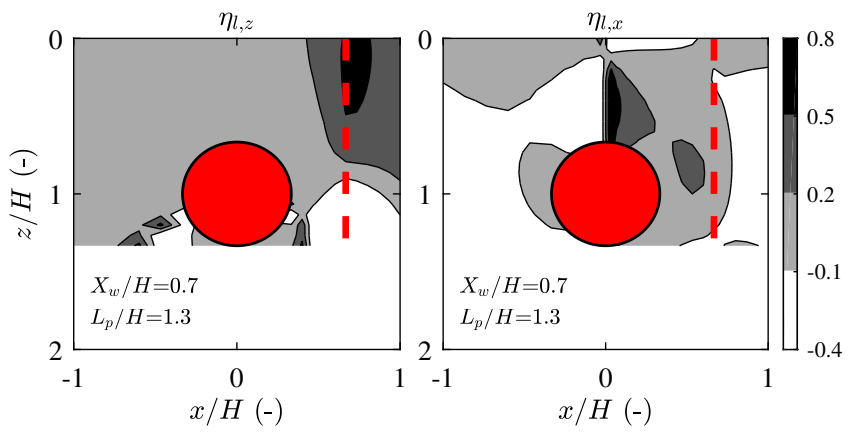

(b)
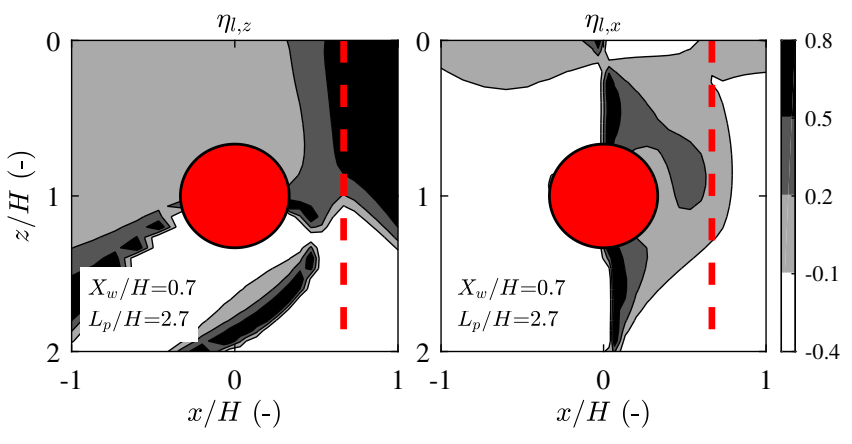

Figure 7. Vertical (left) and horizontal (right) local efficiency $\eta_{l}$ : varying pile location (a vs b) and length (b vs c). 
309

\subsection{Relative soil-to-pile stiffness}

Since the pile stiffening effect leads to the pile shielding mechanism in the elastic solution, it is worthy to quantify the influence of the relative axial/bending pile-soil stiffness; e.g., to know the threshold marking the transition from nearly rigid pile behaviour to semi-flexible and fully-flexible regimes. Results in the previous section (see Figure 4) show that large-diameter concrete pile walls can undergo bending deformations due to horizontal ground movements, while axial compressibility is almost negligible. On the other hand, pile flexibility may be significant for micropiles, hence decreasing their settlement efficiency and leading to a fullyflexible bending behaviour. For instance, Ledesma and Alonso (2017) reported the threshold $\Pi_{4} \approx 0.01$ above which the pile compressibility decreases the wall efficiency $\eta_{w}$.

Because $\Psi$ values should be preferred to $\Pi$ values, the effects of pile stiffness on tunnelwall interactions were also briefly investigated through the influence of $\Psi_{4}$ and $\Psi_{5}$. Figure 8 a plots the wall efficiency $\eta_{w}$ against $\Psi_{4}$ for Case A, with $X_{w} / H=0.7$ and varying $L_{p} / H$. It can be observed that, regardless the normalised pile length, $\Psi_{4} \approx 0.1$ marks the threshold value beyond which pile flexibility decreases the efficiency, whereas a fully-flexible behaviour occurs beyond $\Psi_{4} \approx 100$. Similarly, the influence of bending stiffness on flexural behaviour is investigated, by looking at the pile curvature decrease with respect to a greenfield analysis (or for a fully flexible pile). Figure $8 \mathrm{~b}$ displays the trend of the curvature factor $\omega_{w}$ (i.e., the relative reduction in maximum curvature along the pile) as a function of the relative bending stiffness $\Psi_{5}$. For $\Psi_{5}<1$ a fully rigid behaviour should be expected, while the semi-flexible range extends between $\Psi_{5}=1-10^{5}$.

For brevity, displacement contours and movement profiles for the case of nearly rigid piles are given as supplemental data (Figures S2-S3). Importantly, it can be observed that the difference in horizontal displacement magnitude $u_{x}$ due to a pile stiffness increase is rather limited because of the tilt of the floating piles in homogeneous ground. These supplemental 
data confirmed that limited horizontal efficiency $\eta_{l, x}$ of the barrier should be expected for piles in uniform ground, regardless of their bending stiffness.

(a)

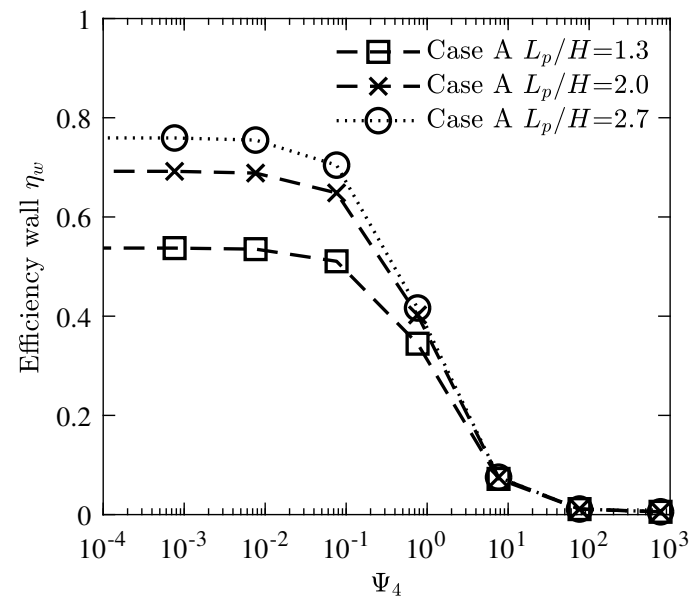

(b)

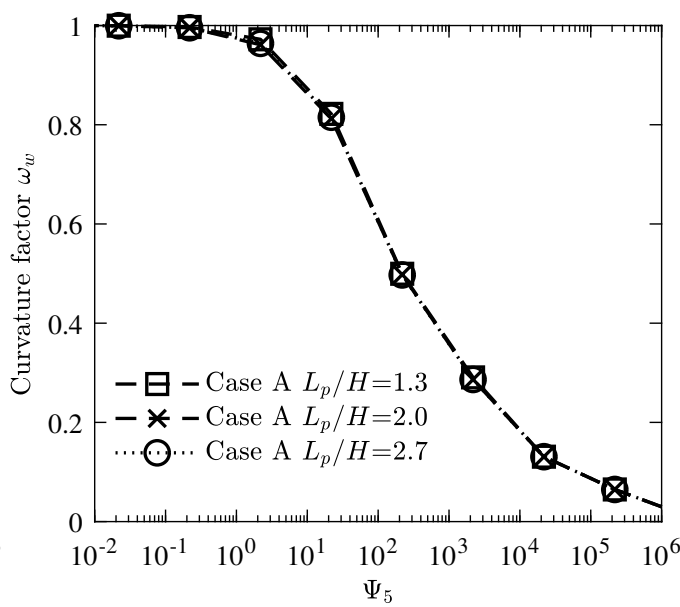

Figure 8. Soil-pile relative stiffness effects: (a) $\eta_{w}$ against $\Psi_{4}$; (b) $\omega_{w}$ against $\Psi_{5}$

\section{Comparison with field data}

\subsection{Line C - Rome}

During the construction of the twin tunnels in contract $\mathrm{T} 3$ of Line $\mathrm{C}$ in Rome, a wall of bored piles was installed in an instrumented area without buildings. The ground conditions at the site are coarse grained made ground (from the surface to $17 \mathrm{~m}$ ), alluvial silty clay and sandy clay (down to $30 \mathrm{~m}$ ), sands and gravels (down to $42 \mathrm{~m}$ ), and stiff clay below (Losacco et al., 2019). The water table depth from ground surface was approximately $9 \mathrm{~m}$. The two EPB tunnels (with $6.71 \mathrm{~m}$ diameters, axis spacing $14.5 \mathrm{~m}$, and depth to tunnel axis $H=25 \mathrm{~m}$ ) were excavated on the east of the pile wall; however, results in this paper only consider the North Tunnel, excavated first and closest to the wall. The barrier consisted of 48 cast-in-situ bored piles with $d_{p}=0.6 \mathrm{~m}$, an embedment length $L_{p}=36 \mathrm{~m}\left(L_{p} / H=1.4\right)$, hence with their

tip in the stiff sandy and gravelly layer, a normalised spacing of $S_{p}=1.5 d_{p}$ and a distance 
from the tunnel centreline $X_{w}=5.2 \mathrm{~m}\left(X_{w} / H=0.2\right)$. Field data (Field) obtained during the excavation and corresponding Class A Finite Element numerical (NUM) results were reported by Losacco et al. (2019), Losacco and Viggiani (2019), and Losacco and Viggiani (2020). In these refined numerical analyses, the pile geometry and location was accurately described by a 3D model, while piles were modelled with elastic solid elements assuming a frictional soil-pile interface. For the tunnelling process, the mechanised excavation and construction process was modelled to consider the head advancement (including, among others, support pressure at the face, shield geometry, grouting of the tail void and its hardening behaviour). Consequently, a hydro-mechanical coupled behaviour of the soil was considered, with different constitutive models for clays and granular materials that were calibrated using information from the geotechnical ground investigation.

Input parameters of the proposed EL model are firstly addressed. A pile row consisting of 48 piles was considered (the numerical analyses had highlighted a dependency on pile number, relating to the barrier length), while pile characteristics were assigned based on the geometry and material properties. Engineering judgement was needed for the selection of the geotechnical parameters. For the ground, a Young's modulus of $150 \mathrm{MPa}$ was assumed considering the small-strain stiffness of the soil along the shaft, a $50 \%$ reduction of its value due to a tunnelling-induced shear strain level of $0.1 \%$, while neglecting that the base layer is stiffer than the ground at shaft. Under these assumptions, $\Psi_{4}=0.4$, indicating a small contribution of the pile shortening to the interaction problem. For the sake of simplicity, Loganathan and Poulos (1998) greenfield displacement field was implemented also for field cases.

The surface settlement profiles predicted by the proposed model (EL), by numerical outcomes (NUM), and by field measurements (Field) are displayed in Figure 9 for both greenfield conditions (GF) and with the pile wall (PW). Two different tunnel volume losses were selected in Figures $9 \mathrm{a}$ and $\mathrm{b} ; V_{l, t}=0.385 \%$ and $0.46 \%$ were selected to match the 
374

centreline greenfield settlement of the numerical model and field data, respectively, for a direct comparison. In Figure 9a, NUM and EL results have similar shapes, both for greenfield movements and considering the tunnel-wall interaction. The mitigating effect decreases with distance from the barrier while a similar asymmetry of the settlement trough is induced, including maximum settlement slightly shifted away from the piles. Importantly, a similar relationship between GF and PW surface settlements was obtained by Rampello et al. (2019) when comparing results for identical tunnel volume losses calculated at the tunnel boundary, as opposed to similar volume loss at the ground surface. However, a slightly greater shielding action of the barrier is predicted by the NUM model than by the EL one, likely due to the stiff ground layer at the pile wall tip (note that the EL model assumes a uniform soil). On the other hand, Figure 9b displays that a greater wall efficiency was measured in the field, making both NUM and EL results conservative.

The subsurface settlements of the NUM and EL models are displayed in Figure 10. Note that isolines of greenfield settlements associated with the L\&P displacement field are slightly wider than for the NUM data (see top charts). Despite this, the impact of the wall on the subsurface soil deformations is comparable, leading to similar decreases of ground settlements with depths on both sides of the wall. Interestingly, the wall action contributes to decreasing settlements both beyond the wall $\left(x>X_{w}\right)$ and above the tunnel centreline $(x=0)$.

\subsection{Line 9 - Barcelona}

Next, the shielding action of the bored pile wall barrier constructed during the excavation of the Line 9 at St Adrià street, in Barcelona, is considered (Di Mariano et al., 2007). This tunnel of $12 \mathrm{~m}$ diameter was executed with a depth $H=23 \mathrm{~m}$ (cover-to-diameter ratio of 1.4) close to multi-storey residential building at an offset of approximately $0.75 \mathrm{H}$. The soil profile is given by: fill ( $3 \mathrm{~m}$ thickness); alluvial sand (11 $\mathrm{m}$ thickness); alluvial silt (6 $\mathrm{m}$ thickness) in which the tunnel crown is located; alluvial sands and gravels (12 m thickness) in which 

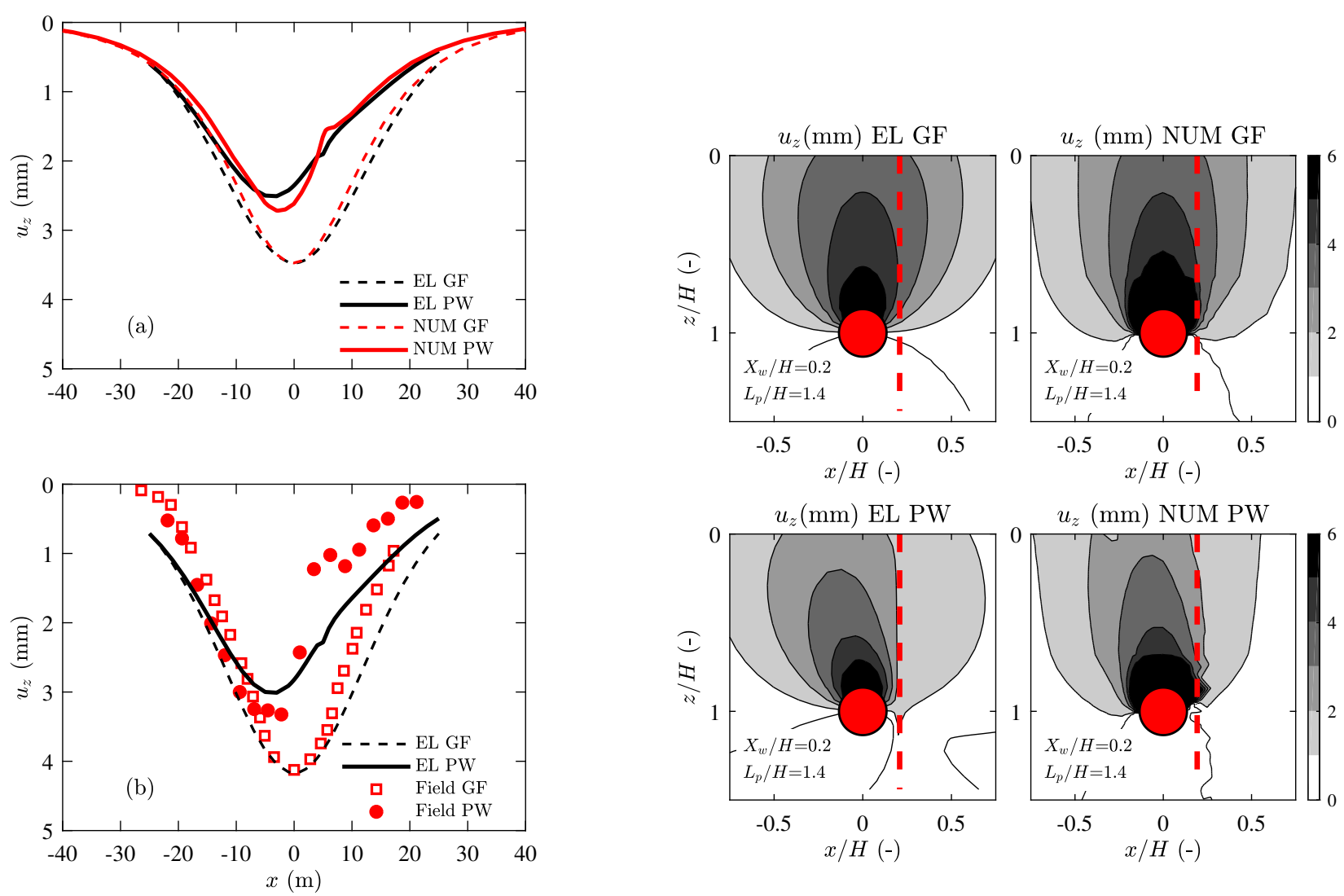

Figure 10. Comparison with the Line

Figure 9. Surface settlements from the Line C field trial Losacco and Viggiani (2020): a) comparison with a) numerical results and b) field data. C field trial: (left) subsurface settlements for the proposed model and $V_{l, t}=0.385 \%$; (right) numerical results from Losacco and Viggiani (2020).

the tunnel springline and invert were located; all overlying a stiff Pleistocene claystone. The water table depth from ground surface was approximately $5 \mathrm{~m}$. Because of this mixed face conditions, the large diameter tunnelling caused significant ground losses $\left(V_{l, t}>1.5 \%\right)$ and settlements. In the model, the barrier was made of 11 bored cast-in-situ piles of $0.65 \mathrm{~m}$ diameter with $L_{p}=29.3 \mathrm{~m}\left(H / L_{p}=1.27\right)$, offset $X_{w}=8.8 \mathrm{~m}\left(X_{w} / H=0.38\right)$, and a normalised pile spacing of $S p / d_{p}=2$.

Di Mariano et al. (2007) reported settlements measured in the presence of the wall (Field PW) as well as back-calculated 2D numerical results (NUM) both in greenfield conditions (GF) and with the wall model activated (PW). In these back-analyses, the layered soil was 


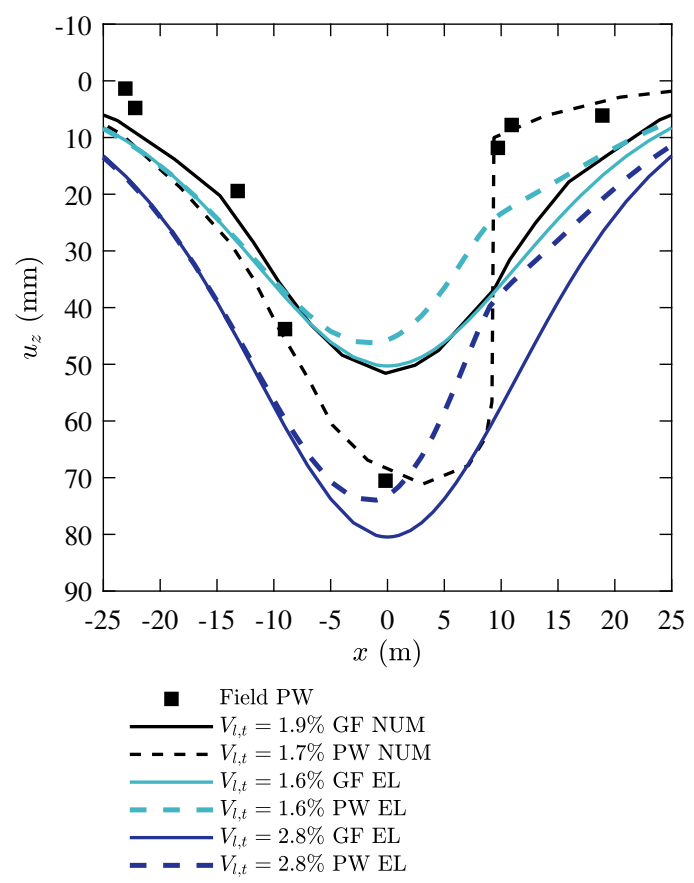

Figure 11. Comparison with the Barcelona monitoring (Di Mariano et al., 2007): surface settlements.

modelled with a Mohr-Coulomb constitutive law and elastic beam elements were used for the wall. Tunnelling was modelled with the stress relief method.

Figure 11 compares the EL surface settlement predictions with field measurements and with numerical results. Two tunnel volume losses were used in the EL solution $\left(V_{l, t}=1.6 \%\right.$ and $2.8 \%$ ); with values selected to replicate the numerical greenfield results and the field settlements, respectively. For the ground, $E_{s}=100 \mathrm{MPa}$ was considered. Although the EL solutions are conservative, their predicted shape matches neither the field data nor the numerical results. As noted from the numerical results (NUM PW), surface settlements were greater than for the back-calculated greenfield profile (NUM GF), while settlement reductions induced by the pile barrier was significant, leading to a maximum settlement behind the wall of approximately $11.8 \mathrm{~mm}$ rather than $36 \mathrm{~mm}$. The numerical efficiency of the wall $\eta_{w}=67 \%$ is greater than the EL efficiency $35 \%$. 
There are several causes for the differences between NUM and EL results, discussed considering the numerical work of Rampello et al. (2019). Firstly, the poor performance of the large $12 \mathrm{~m}$ diameter tunnel boring machine that resulted in a rather large tunnel volume loss of $V_{l, t}=1.6 \%$ and, thus, in large settlements. Secondly, the pile wall was modelled by Di Mariano et al. (2007) using a 2D diaphragm, rather than a pile wall. As discussed by Rampello et al. (2019) a 2D diaphragm wall prevents the tunnel ground loss from propagating beyond the wall, hence shielding the soil beyond it from the ground loss; however, this shielding effect is minor for the pile walls simulated by Rampello et al. (2019). Thirdly, this settlement level led to soil negative friction that (likely) exceeded the ultimate shaft friction of the wall. All points could contribute to the 2D numerical results from Di Mariano et al. (2007) displaying a settlement discontinuity between the two sides of the wall. Despite uncertainties on the field shape of the surface settlements, these results from the Line 9 highlighted the limitations of the proposed elastic approach EL in predicting the wall efficiency for relatively high volume losses.

\section{Discussion and considerations on the model applicability}

The first case study indicated that a reasonable estimate of the subsurface settlements on both sides of the wall is possible for small ground deformations (associated with small tunnel volume loss). The second case illustrated that conservative estimates of the wall efficiency would be provided by elastic methods for large tunnelling-induced ground losses, although the displacement distribution due to the pile wall may not be well predicted by the elastic solution (because of the soil-pile compatibility assumption is not fulfilled). Unfortunately, field evidence on displacements shapes and settlement discontinuities at the pile wall sides are not conclusive.

As a preliminary step to advanced modelling, there is scope in practice for using versatile 
444

EL 3D models in risk assessments. Although numerical predictions obtained from advanced numerical modelling were satisfactory in both cases, these simulations were computationally expensive and needed a careful calibration using geotechnical investigations to produce an advanced ground model with an elasto-plastic interface. The obtained EL 3D predictions have a good level of fidelity when tunnelling results in small volume losses and millimetrelevel displacements, i.e. when the assumptions of linear elastic ground behaviour and perfect pile-soil compatibility are reasonable. For large tunnelling-induced movements, the wall efficiency is under-predicted while only the shape of the soil movements beyond the wall may be considered representative.

To illustrate the reliability and limitations of the proposed 3D EL model, wall efficiency predictions as a function of the pile length-to-tunnel depth ratio are compared with an extensive dataset covering a variety of ground conditions. Figure 12 displays the predictions of the wall efficiency values of the proposed PW EL model (black solid lines), against field data (Di Mariano et al., 2007; Losacco et al., 2019; Nikiforova and Vnukov, 2012), numerical outcomes (Rampello et al., 2019; Losacco and Viggiani, 2020; Bai et al., 2014) and centrifuge results (Bilotta et al., 2006) of pile walls (black markers). All PW results are for a pile spacing $S_{p} / d_{p} \leq 3$. Also, the 2D EL model results of Ledesma and Alonso (2017) (light coloured line) is plotted against the results (light coloured markers) for frictional diaphragm walls (DW) obtained numerically by Rampello et al. (2019) and from field monitoring by Nikiforova and Vnukov (2012). For the 3D EL model, results from the tunnel Case A in Table 1 are considered.

First, the introduced case studies are briefly described. Bai et al. (2014) modelled the efficiency of a pile wall ( $0.8 \mathrm{~m}$ diameter bored piles with spacing $s / d=1.5)$ embedded in the Shanghai silty and clayey soil; numerical predictions of tunnelling-induced pile settlements agreed with field measurements. Nikiforova and Vnukov (2012) measured, for tunnelling in clay overlain by sand in Moscow, induced settlements next to a pile wall consisting of $16 \mathrm{~m}$ 
long steel pipes $\left(d_{p}=0.16 \mathrm{~m}\right.$ and spacing of $\left.S_{p} / d_{p}=1.5\right)$ and to a diaphragm wall consisting of two rows of $1.2 \mathrm{~m}$ deep-mix secant piles). Bilotta et al. (2006) carried out centrifuge tests of tunnelling adjacent to a row of aluminium micropiles (at prototpye scale, $d_{p}=0.26 \mathrm{~m}$ and spacing of $S_{p} / d_{p}=3$ ) driven in Speswhite kaolin. Rampello et al. (2019) simulated floating pile walls and continuous diaphragms embedded in a ground with a stratigraphy similar to the field trial of the Line $\mathrm{C}$.

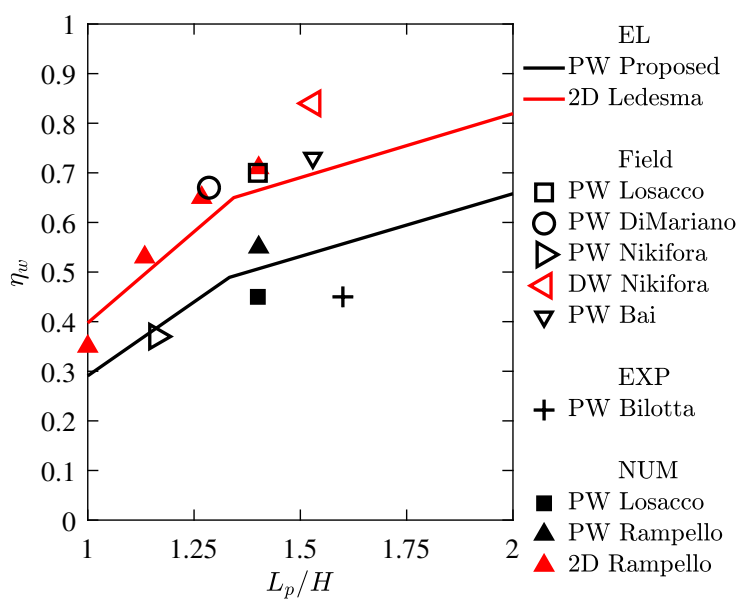

Figure 12. Local efficiency $\eta_{w}$ against $L_{p} / H$ for pile wall (PW) and diaphragm (DW): elasticity solutions (EL) against field (Field), experimental (EXP) and numerical (NUM) data.

Next, results in Figure 12 are discussed. Despite the wall efficiency being obtained for different $\Psi$ values (for instance, different offsets), the agreement between elastic predictions and numerical/experimental results is good for both pile and diaphragm wall barriers, except for a few specific cases, confirming that $L_{p} / H$ plays a major role in determining $\eta_{w}$ (as already suggested by Figure 5). In fact, Figure 12 shows that both EL 3D results for pile walls PW underpredict the wall efficiency inferred from field data of Bai et al. (2014), Di Mariano et al. (2007), and Losacco et al. (2019), which are interestingly close to 2D efficiency predictions. This underestimation is partly due to a stiff soil layer at the pile tip, that restrains the pile from settling, thus increasing the pile efficiency; the uniform soil stiffness used by the proposed model does not capture this additional mechanism. On the other hand, the EL 3D 
486

trend does agree with pile wall PW numerical results of Losacco et al. (2019), field data of Nikiforova and Vnukov (2012), and centrifuge outcomes of Bilotta et al. (2006).

Finally, the potential use of the model for more complex scenarios is also discussed. The main advantages of the 3D solutions (with respect to $2 \mathrm{D}$ models) are: (i) that it allows as input 2D and 3D displacement fields (e.g. due to tunnel advancement), (ii) that it rationally models any pile wall configuration (e.g. varying longitudinal spacing between piles, unaligned piles), and (iii) that, with future extensions, it can consider varying stiffness along the pile length.

\section{Conclusions}

In this paper, a three-dimensional linear elastic two-stage continuum-based model for tunnelpile group interaction was used to study the protective action of barriers next to new tunnel excavations. This model, relying on a limited number of geometrical and elasticity parameters, can be used as a quick preliminary step to advanced modelling while allowing to consider any greenfield displacement input. Analyses were carried out to evaluate the capabilities of pile walls in decreasing the vertical and horizontal movements of the ground thanks to their axial and bending stiffness. The following main conclusions can be drawn.

- The presented three-dimensional elastic model allows estimating the shielding action of a pile wall barrier against tunnelling-induced settlements and horizontal movements, with the latter being not studied by previous empirical and analytical models. More importantly, the displacement field within the entire ground mass resulting from the tunnel-barrier interaction is directly obtained by solving the interaction problem, overcoming the limitations of previous methods only describing the reduction in settlements at the surface or pile heads. This displacement field can be used for risk assessments of existing surface or subsurface structures. 
- For this tunnel-pile wall interaction model, it was shown that vertical and horizontal displacements at any point can be obtained, which can be used as input for uncoupled and coupled soil-structure interaction models. Results illustrated how the stiffness of the pile wall barrier modifies both surface and subsurface ground movements, confirming the results of previous analytical solutions for diaphragm walls (i.e., in plane-strain conditions).

- Dimensionless displacements and local efficiency values at surface and subsurface locations were illustrated. Plots in terms of displacement curves and contours may be used for preliminary assessments.

- Validation against a field-trial data and numerical Class A predictions confirmed the robustness of the proposed elastic approach when small-to-medium tunnelling-induced volume losses are induced (giving a small-strain regime for the soil). Further work is needed to improve the model predictions at large settlement levels.

- Field data reported pile wall efficiency often greater than the values predicted both by this elastic model and more numerical advanced simulations. Future work will need to provide insights into this discussion, considering the influence of layered ground conditions with different properties at each layer.

\section{Acknowledgements}

This project has received funding from the European Union's Horizon 2020 research and innovation programme under the Marie Sklodowska-Curie grant agreement No 793715. 


\section{NOTATION}

$d_{p} \quad$ pile diameter

$i \quad$ offset of the greenfield inflection point

$u_{\text {norm }}$ displacement as normalised ground loss

$u_{x} \quad$ horizontal displacement

$u_{z} \quad$ vertical displacement

$x \quad$ transverse horizontal distance

y longitudinal horizontal distance

$z \quad$ depth

$A_{p} \quad$ pile cross-sectional area

C cover

D tunnel diameter

$I_{p} \quad$ pile second moment of area

$E_{p} \quad$ pile Young's modulus

$E_{s} \quad$ soil Young's modulus

$H \quad$ depth of the new tunnel

$L_{p} \quad$ pile wall length

$R \quad$ tunnel radius

$S_{P} \quad$ longitudinal pile spacing

$V_{0} \quad$ tunnel area

$V_{l, t} \quad$ percentage tunnel volume loss

$X_{w} \quad$ pile wall offset

$\eta_{l, x}$ local efficiency in horizontal movement reduction

$\eta_{l, z} \quad$ local efficiency in vertical movement reduction

$\eta_{w} \quad$ wall efficiency in settlement reduction

$\chi \quad$ curvature

$\epsilon \quad$ volumetric term

$\omega_{w} \quad$ wall efficiency in curvature reduction

$\nu_{s} \quad$ soil Poisson's ratio of the soil

$\rho \quad$ ovalization term

$\xi \quad$ corrective term

$\Delta V \quad$ ground loss

$\Pi_{i} \quad i$ th dimensionless group $\Pi$

$\Psi_{i} \quad i$ th dimensionless group $\Psi$ $\mathbf{f}_{\mathrm{w}}$ interaction force vector at the pile locations

$\mathbf{k}_{s} \quad$ ground stiffness matrix at the pile locations

$\mathbf{k}_{w} \quad$ stiffness matrix of the pile group

$\mathbf{u}_{w} \quad$ displacement vector of the pile group

$\mathbf{u}_{w}^{g f} \quad$ greenfield displacement vector at the pile locations

$\mathbf{F}_{w} \quad$ interaction force vector at the ground locations

$\mathbf{L}_{s} \quad$ soil flexibility matrix at the ground locations

$\mathbf{U}_{s} \quad$ final displacement vector of the ground locations

$\mathbf{U}_{s}^{g f} \quad$ greenfield displacement vector of the ground

$\mathbf{U}_{s}^{i n t}$ interaction displacement vector of the ground

GF greenfield

PW pile wall

DW diaphragm wall

NUM numerical

EL elasticity

EMP empirical

EXP experimental

$\mathrm{P} \quad$ pile

S soil

SA semi-analytical

TBI tunnel-barrier interaction

TSI tunnel-structure interaction

W wall 


\section{References}

Bai, Y., Yang, Z., Jiang, Z., 2014. Key protection techniques adopted and analysis of influence on adjacent buildings due to the Bund Tunnel construction. Tunnelling and Underground Space Technology 41, 24-34. doi:10.1016/j.tust.2013.11.005.

Basile, F., 2014. Effects of tunnelling on pile foundations. Soils and Foundations 54, 280-295. doi:10.1016/j.sandf.2014.04.004.

Bilotta, E., Bitetti, B., McNamara, A.M., Taylor, R.N., 2006. Micropiles to reduce ground movements induced by tunnelling, in: Ng, C.W.W., Zhang, L.M., Wang, Y.H. (Eds.), Proceedings of the 6th International Conference on Physical Modelling in Geotechnics - Physical Modelling in Geotechnics - 6th ICPMG '06, Taylor \& Francis, London, Hong Kong. pp. 1139-1144.

Bilotta, E., Russo, G., 2011. Use of a line of piles to prevent damages induced by tunnel excavation. Journal of Geotechnical and Geoenvironmental Engineering 137, 254-262. doi:10.1061/(ASCE) GT.1943-5606.0000426.

Chen, L.T., Poulos, H.G., Loganathan, N., 1999. Pile responses caused by tunneling. Journal of Geotechnical and Geoenvironmental Engineering 125, 207-215. doi:10.1061/(ASCE) 1090-0241 (1999) 125:3(207).

Di Mariano, A., Gesto, J.M., Gens, A., Schwarz, H., 2007. Ground deformation and mitigating measures associated with the excavation of a new Metro line, in: Cuéllar, V., Dapena, E., Alonso, E., Echave, J.M., Gens, A., De Justo, J.L., Oteo, C., Rodríguez-Ortiz, J.M., Sagaseta, C., Sola, P., Soriano, A. (Ed.), Proceedings of the XIV European Conference on Soil Mechanics and Geotechnical Engineering, ECSMGE, IOS Press, Amsterdam, The Netherlands, Madrid, Spain. pp. 1901-1906.

Franza, A., Acikgoz, S., DeJong, M.J., 2020. Timoshenko beam models for the coupled analysis of building response to tunnelling. Tunneling and Underground Construction 96, 103160. doi:10 . $1016 / j$.tust. 2019.103160.

Franza, A., Marshall, A.M., Jimenez, R., 2019a. Elastic analysis of tunnelling beneath capped pile groups, in: Sigursteinsson, H., Erlingsson, S., Bessason, B. (Eds.), Proceedings of the XVII ECSMGE-2019: Geotechnical Engineering foundation of the future, Icelandic Geotechnical Society, Reykjavík, Iceland. doi:10.32075/17ECSMGE-2019-0529.

Franza, A., Marshall, A.M., Jimenez, R., 2019b. Nonlinear soil-pile interaction induced by ground settlements: pile displacements and internal forces. Géotechnique In Press doi:10.1680/jgeot. 19.P. 078 .

González, C., Sagaseta, C., 2001. Patterns of soil deformations around tunnels. Application to the extension of Madrid Metro. Computers and Geotechnics 28, 445-468. doi:10.1016/ S0266-352X (01) 00007-6.

Harris, D.I., 2001. Protective measures, in: Building response to tunnelling: Case studies from construction of the Jubilee Line Extension, London. Thomas Telford Publishing, pp. 135-176.

Ledesma, A., Alonso, E.E., 2017. Protecting sensitive constructions from tunnelling: the case of World Heritage buildings in Barcelona. Géotechnique 67, 914-925. doi:10.1680/jgeot. SiP17. P.155. 
Loganathan, N., Poulos, H.G., 1998. Analytical prediction for tunneling-induced ground movements in clays. Journal of Geotechnical and Geoenvironmental Engineering 124, 846-856. doi:10.1061/ (ASCE) 1090-0241 (1998) 124: 9 (846).

Loganathan, N., Poulos, H.G., Xu, K.J., 2001. Ground and pile-group responses due to tunnelling. Soils and Foundations 41, 57-67.

Losacco, N., Romani, E., Viggiani, G., Di Mucci, G., 2019. Embedded barriers as a mitigation measure for tunnelling induced settlements: A field trial for the Line $\mathrm{C}$ in Rome, in: Peila, D., Viggiani, G., Celestino, T. (Eds.), Proceedings of the WTC 2019 ITA-AITES World Tunnel Congress - Tunnels and Underground Cities: Engineering and Innovation meet Archaeology, Architecture and Art. CRC Press/Balkema, Leiden, The Netherlands, Naples, Italy, pp. 58455854. doi:10.1201/9780429424441-618.

Losacco, N., Viggiani, G.M., 2019. Class A prediction of mechanised tunnelling in Rome. Tunnelling and Underground Space Technology 87, 160-173. doi:10.1016/j.tust.2019.02.020.

Losacco, N., Viggiani, G.M.B., 2020. Mechanised Tunnel Excavation Through an Instrumented Site in Rome: Class A Predictions and Monitoring Data. Lecture Notes in Civil Engineering 40, 245-254. doi:10.1007/978-3-030-21359-6_26.

Mair, R.J., Taylor, R.N., 1997. Theme lecture: Bored tunnelling in the urban environment, in: 14th International conference on soil mechanics and foundation engineering, Balkema, Hamburg. pp. $2353-2385$.

Mair, R.J., Taylor, R.N., Bracegirdle, A., 1993. Subsurface settlement profiles above tunnels in clay. Géotechnique 43, 315-320. doi:10.1680/geot.1993.43.2.315.

Mair, R.J., Taylor, R.N., Burland, J.B., 1996. Prediction of ground movements and assessment of risk of building damage due to bored tunnelling, in: Mair, R.J., Taylor, R.N. (Eds.), Proceedings of the International Symposium on Geotechnical Aspects of Underground Construction in Soft Ground, Balkema, Rotterdam, London, United Kingdom. pp. 713-718.

Nikiforova, N., Vnukov, D., 2012. Geotechnical cut-off diaphragms for built-up area protection in urban underground development, in: Viggiani, G. (Ed.), Proceedings of the 7th International Symposium on Geotechnical aspects of underground construction in soft ground, CRC Press, Leiden, The Netherlands, Rome, Italy. pp. 927-932.

Pender, M.J., 1980. Elastic solutions for a deep circular tunnel. Géotechnique 30, 216-222. doi:10. $1680 /$ geot $.1980 .30 \cdot 2.216$.

Pinto, F., Whittle, A.J., 2006. Discussion of "Elastic solution for tunneling-induced ground movements in clays" by K. H. Park. International Journal of Geomechanics 6, 72-73. doi:10.1061/ (ASCE) 1532-3641(2006)6:1(72).

Pinto, F., Whittle, A.J., 2014. Ground Movements due to Shallow Tunnels in Soft Ground. I: Analytical Solutions. Journal of Geotechnical and Geoenvironmental Engineering 140, 04013040. doi:10.1061/(ASCE) GT.1943-5606.0000948.

Pinto, F., Zymnis, D.M., Whittle, A.J., 2014. Ground Movements due to Shallow Tunnels in Soft Ground. II: Analytical Interpretation and Prediction. Journal of Geotechnical and Geoenvironmental Engineering 140, 1-11. doi:10.1061/(ASCE) GT.1943-5606.0000947. 
611

612

613

614

615

616

617

618

619

620

621

622

623

624

625

Potts, D.M., Addenbrooke, T.I., 1997. A structure's influence on tunnelling-induced ground movements. Proceedings of the ICE - Geotechnical Engineering 125, 109-125. doi:10.1680/igeng. 1997.29233.

Rampello, S., Fantera, L., Masini, L., 2019. Efficiency of embedded barriers to mitigate tunnelling effects. Tunnelling and Underground Space Technology 89, 109-124. doi:10.1016/j . tust. 2019. 03.027.

Sagaseta, C., 1987. Analysis of undrained soil deformation due to ground loss. Géotechnique 37, 301-320. doi:10.1680/geot.1987.37.3.301.

Song, G., 2019. The use of protective structures to reduce tunnelling induced damage to buildings. Ph.D. Thesis, Nottingham University, Department of Civil Engineering, Nottingham .

Verruijt, A., Booker, J., 1996. Surface settlements due to deformation of a tunnel in an elastic half plane. Géotechnique 46, 753-756. doi:10.1680/geot.1996.46.4.753.

Zhang, P., Yin, Z.Y., Chen, R.P., 2020. Analytical and Semi-Analytical Solutions for Describing Tunneling-Induced Transverse and Longitudinal Settlement Troughs. International Journal of Geomechanics 20, 04020126. doi:10.1061/(ASCE) GM.1943-5622.0001748. 